Preprints of the

Max Planck Institute for

Research on Collective Goods

Bonn 2010/10

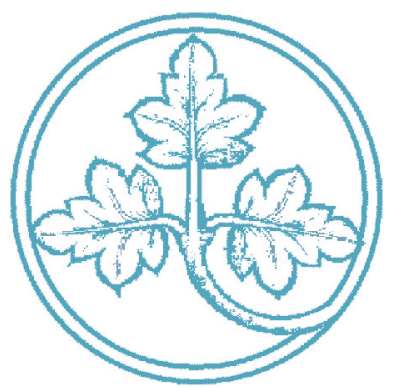

Braucht die Rechtswissenschaft eine empirische Wende?

Niels Petersen

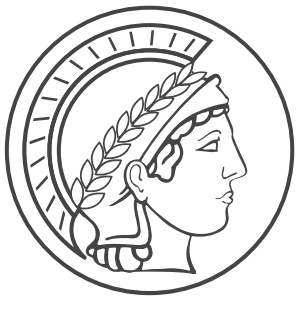




\section{Braucht die Rechtswissenschaft eine empirische Wende?}

Niels Petersen

April 2010 


\title{
Braucht die Rechtswissenschaft eine empirische Wende?
}

\author{
von Niels Petersen*
}

\section{Einleitung}

In der Rechtswissenschaft werden normative und empirische Fragen in der Regel deutlich voneinander getrennt. Die Frage nach dem, was ist, ist von derjenigen, was sein soll, zu unterscheiden. Dies lernt der Jurist schon im frühen Stadium seiner Ausbildung, wenn er den Dreischritt der Gesetzesanwendung kennenlernt. ${ }^{1}$ In diesem nimmt er immer erst die Definition des gesetzlichen Tatbestandsmerkmals vor, beschäftigt sich also mit dem normativen Teil, in dem das Sollen bestimmt wird. Erst im zweiten Schritt folgt der tatsächliche Teil, die Feststellung der Wirklichkeit und der Abgleich des Vorgefundenen mit der Definition. Der wissenschaftliche Diskurs in der Rechtswissenschaft beschäftigt sich dabei ganz überwiegend mit dem normativen Teil ${ }^{2}-$ die Feststellung der Wirklichkeit ist letztlich Sache des Richters. Auch der empirische Fokus der Praxis ist allerdings beschränkt. Der Richter beschäftigt sich vor allem mit der Sachverhaltsaufklärung im Einzelfall und hat einen eher beschreibenden Zugang zur Wirklichkeit. Dagegen ist die Beschäftigung mit sozialwissenschaftlichen Gesetzmäßigkeiten, mit allgemeinen kausalen Zusammenhängen zwischen zwei sozialen Phänomenen sowohl in der juristischen Praxis als auch in der Rechtswissenschaft bisher nicht sonderlich stark ausgeprägt.

Dies ist nicht in allen Rechtskreisen der Fall. So gibt es etwa in den Vereinigten Staaten schon seit längerem eine Bewegung, die sich mit empirical legal studies, also empirischer Rechtswissenschaft beschäftigt und dabei vor allem gesellschaftliche Phänomene und soziale GesetzmäBigkeiten in den Blick nimmt. ${ }^{3}$ Diese stärkere empirische Fokussierung in den USA ist zum Teil mit der Ausrichtung der dortigen Rechtswissenschaft zu erklären, die sich stärker mit rechtssoziologischen Fragen oder Fragen der Rechtspolitik beschäftigt als mit der Frage nach der Ausle-

\footnotetext{
* Dr. iur. Columbia University, New York, und Max-Planck-Institut zur Erforschung von Gemeinschaftsgütern, Bonn. Der Beitrag beruht auf einem Vortrag, den der Verfasser im Rahmen des Karlsruher Dialogs zum Informationsrecht am 12. Januar 2010 in Karlsruhe gehalten hat. Herzlicher Dank gilt Carsten Burhop, Christoph Engel und Emanuel Towfigh für vielfältige Anregung und Kritik.

1 S. dazu Karl Larenz, Methodenlehre der Rechtswissenschaft, 6. Aufl. 1991, S. 271 ff.

2 Es gibt jedoch vereinzelte Studien, die schon früh versucht haben, empirische Forschung für die dogmatische Rechtswissenschaft fruchtbar zu machen, s. etwa Gunther Teubner, Standards und Direktiven in Generalklauseln, 1971. In jüngerer Zeit versucht vor allem die rechtsökonomische Literatur sozialwissenschaftliche Erkenntnisse in die juristische Argumentation einzuführen, s. vor allem Anne van Aaken, „Rational Choice“ in der Rechtswissenschaft. Zum Stellenwert der ökonomischen Theorie im Recht, 2003. Allerdings nimmt diese oft mehr auf analytische Modelle als auf empirische Erkenntnisse Bezug.

3 S. Michael Heise, The Past, Present, and Future of Empirical Legal Scholarship: Judicial Decision Making and the New Empiricism, U. Ill. L. Rev. 2002, 819; Frank Cross/Michael Heise/Gregory C. Sisk, Above the Rules: A Response to Epstein and King, U. Chi. L. Rev. 69 (2002), 135; Richard L. Revesz, A Defense of Empirical Legal Scholarship, U. Chi. L. Rev. 69 (2002), 169.
} 
gung des geltenden Rechts, die in Deutschland ganz überwiegend im Zentrum der Betrachtung steht. $^{4}$

Das bedeutet jedoch nicht, dass die empirische Sozialwissenschaft für den Juristen, der sich mit der Rechtsauslegung beschäftigt, belanglos ist. Vielmehr soll in diesem Beitrag herausgearbeitet werden, dass Empirie und Normativität in der juristischen Argumentation sehr viel enger miteinander verschränkt sind, als es das klassische Subsumtionsmodell nahelegt. Insofern hat die Beschäftigung mit empirischer Forschung für die Rechtswissenschaft auch dann Relevanz, wenn diese sich vor allem mit der Auslegung des geltenden Rechts und damit mit normativen Fragestellungen beschäftigt. Die These wird im Folgenden in zwei Schritten begründet. Zunächst soll dargelegt werden, dass alle normativen Modelle zu einem gewissen Grade empirisch bedingt sind und dies auch und gerade für die Rechtswissenschaft gilt. In einem zweiten Schritt soll dann vor zwei Fehlschlüssen gewarnt werden. Zum einen ist das juristische Instrumentarium allein nicht geeignet, soziale Tatsachen angemessen zu beurteilen. Zum anderen kann die empirische Arbeit nicht einfach an Sozialwissenschaftler delegiert werden, weil empirische Studien ihrerseits häufig auch normative Implikationen haben.

\section{Die empirische Bedingtheit normativer Modelle}

\section{Normativität und Wirklichkeit}

In der Theorie sind Sollen und Sein sind grundsätzlich voneinander getrennte Sphären. Wir können weder vom Sein auf das Sollen schließen, noch vom Sollen auf das Sein. Ersteres ist der sogenannte naturalistische Fehlschluss. ${ }^{5}$ Es lässt sich nicht allein aus der Beobachtung eines tatsächlichen Zustandes oder Geschehens darauf schließen, dass das, was beobachtet wird, auch normativ richtig oder wertvoll ist. Allein aus der Beobachtung, dass sich in der Natur regelmäßig der Stärkere durchsetzt, können wir noch kein Recht des Stärkeren ableiten. Auch umgekehrt passiert es manchmal, dass aus Sollenssätzen auf das Sein geschlossen wird - ein normativistischer Fehlschluss, der gerade in der Juristerei nicht unbekannt ist. ${ }^{6}$

Wenn wir weder unmittelbar vom Sein auf das Sollen noch vom Sollen auf das Sein schließen können, bedeutet dies umgekehrt aber nicht, dass es sich bei beiden um vollkommen getrennte Sphären handelt. Vielmehr sind sie in doppelter Weise miteinander verzahnt. Wenn wir davon ausgehen, dass unser Wissen über die Welt nicht nur aus Erfahrung geformt wird, sondern auch durch unsere Perspektive geprägt wird, mit der wir die Welt betrachten, ${ }^{7}$ dann haben unsere

4 S. dazu Kristoffel Grechenig/Martin Gelter, Divergente Evolution des Rechtsdenkens - Von amerikanischer Rechtsökonomie und deutscher Dogmatik, RabelsZ 72 (2008), 513.

$5 \quad$ Grundlegend George Edward Moore, Principia Ethica, 1959 [1903], S. 62.

6 Vgl. Anne van Aaken, Funktionale Rechtswissenschaftstheorie für die gesamte Rechtswissenschaft, in: Matthias Jestaedt/Oliver Lepsius (Hg.), Rechtswissenschaftstheorie, 2008, 79, 88, die in der Rechtswissenschaft die Gefahr des normativistischen Fehlschlusses für größer hält als die des naturalistischen Fehlschlusses.

7 S. nur Hans-Georg Gadamer, Wahrheit und Methode. Grundzüge einer philosophischen Hermeneutik, 1960, S. 261-69; Jürgen Habermas, Erkenntnis und Interesse, 1968, S. 116-43; Charles Taylor, Interpretation and the Sciences of Man, The Review of Metaphysics 25 (1971), 3, 10-17. 
normativen Vorstellungen einen Einfluss darauf, was wir tatsächlich beobachten. Umgekehrt setzen normative Vorstellungen immer auch die faktische Kenntnis der zu regelnden Situation voraus. Man kann nur sinnvoll entscheiden, was sein soll, wenn man weiß, was überhaupt tatsächlich möglich ist. Unabhängig davon unterliegen normative Modelle zudem immer bestimmten empirischen Voraussetzungen. Ein normatives Postulat mag noch so elegant und attraktiv sein - wenn es von Voraussetzungen ausgeht, die unserem Wissen über die Wirklichkeit widersprechen, dann kann es für die Praxis wertlos sein. Dies soll im Folgenden an zwei Beispielen erläutert werden.

So ging etwa Jean-Jacques Rousseau in seinem Gesellschaftsvertrag noch davon aus, dass die Demokratie nur den Göttern vorbehalten sei:

„[w]enn es ein Volk von Göttern gäbe, würde es sich demokratisch regieren. Eine so vollkommene Regierungsform entspricht den Menschen nicht.“ 8

Auch wenn wir heute Rousseau in seiner Einschätzung nicht mehr zustimmen würden, so zeigt es doch, dass das Funktionieren von Demokratie auf bestimmten Annahmen über die Natur des Menschen zu beruhen scheint. Es vertritt zwar niemand mehr ernsthaft, dass der Mensch grundsätzlich demokratieunfähig ist. Allerdings gibt es eine lebhafte sozialwissenschaftliche Diskussion zu der Frage, ob das Funktionieren von Demokratie nicht von bestimmten sozio-ökonomischen Voraussetzungen, wie etwa einem gewissen Grad an wirtschaftlicher Entwicklung 9 , sozialer und ethnischer Homogenität ${ }^{10}$ oder gar dem kulturellen Hintergrund ${ }^{11}$ abhängt.

Geht man davon aus, dass Demokratie nur unter bestimmten sozialen, ökonomischen oder kulturellen Umständen stabil sein kann, dann verliert das Modell an Attraktivität, wenn diese Umstände nicht gegeben sind. ${ }^{12}$ Ansonsten würden wir vom normativ Wünschenswerten auf das Gegebene schließen - der oben identifizierte normativistische Fehlschluss. Selbst wenn man aber davon ausgeht, dass Demokratie unter allen Umständen möglich ist, wird zumindest die konkrete Ausgestaltung des institutionellen Designs von den tatsächlichen Voraussetzungen abhängen. ${ }^{13}$ Die ideale Institutionenordnung sieht in einem Vielvölkerstaat anders aus als in einer homogenen Kulturnation. ${ }^{14}$

8 Jean-Jacques Rousseau, Vom Gesellschaftsvertrag oder Grundlagen des politischen Rechts, 1996 [1762], S. 93 (Übersetzt von Erich Wolfgang Skwara) („S'il y avait un peuple de Dieux, il se gouvernerait démocratiquement. Un gouvernement si parfait ne convient pas à des hommes.“).

9 Seymour Martin Lipset, Some Social Requisites of Democracy: Economic Development and Political Legitimacy, Am. Pol. Sc. Rev. 53 (1959), 69; Kenneth A. Bollen/Robert W. Jackman, Economic and Noneconomic Determinants of Political Democracy in the 1960s, Res. Pol. Soc. 1 (1985), 27; Larry Diamond, Economic Development and Democracy Reconsidered, in: Gary Marks/Larry Diamond (Hg.), Reexamining Democracy. Essays in Honor of Seymour Martin Lipset, 1992, 93; Adam Przeworski/Michael E. Alvarez/José Antonio Cheibub/Fernando Limongi, Democracy and Development: Political Institutions and WellBeing in the World 1950-1990, 2000.

10 Grundlegend John Stuart Mill, Considerations on Representative Government, 1861.

11 Samuel P. Huntington, The Clash of Civilizations?, Foreign Aff. 72 (1993), 22.

$12 \mathrm{Zu}$ den normativen Folgerungen, die daraus für das Völkerrecht zu ziehen sind, s. Niels Petersen, Demokratie als teleologisches Prinzip - Zur Legitimität von Staatsgewalt im Völkerrecht, 2009.

13 Giovanni Sartori, Demokratietheorie, 2006, S. 16.

14 S. dazu insbesondere Arend Lijphart, Democracy in Plural Societies, 1977. 
Ein weiteres Beispiel einer empirisch bedingten normativen Theorie ist die demokratietheoretische Variante der Diskurstheorie. Die Schule der deliberativen Demokratie stellt den rationalen Diskurs ins Zentrum ihrer Theorie. ${ }^{15}$ Sie geht davon aus, dass bei politischen Entscheidungen nicht in erster Linie die numerische Mehrheit der Köpfe, sondern die Kraft der in der Diskussion vorgebrachten Argumente entscheiden solle:

„Die deliberative Politik gewinnt ihre legitimierende Kraft aus der diskursiven Struktur einer Meinungs- und Willensbildung, die ihre sozialintegrative Kraft nur dank der Erwartung einer vernünftigen Qualität ihrer Ergebnisse erfüllen kann. “16

Das entscheidende Argument für die deliberative Demokratie ist also, dass wir vom deliberativen Prozess bessere Ergebnisse erwarten können als von einer bloßen Mehrheitsentscheidung ohne vorherigen argumentativen Austausch. Die empirische Annahme dabei ist, dass der Diskurs tatsächlich zu besseren Kollektiventscheidungen führt. Dies ist jedoch nicht selbstverständlich. So legt die sozialpsychologische Forschung nahe, dass Diskussionen innerhalb einer Gruppe unter bestimmten Umständen zu schlechteren Ergebnissen führen, als wenn man einfach nach Köpfen abgestimmt hätte. ${ }^{17}$ So elegant das normative Modell also auch ist, unterliegt es doch gewissen empirischen Voraussetzungen, die es zu berücksichtigen gilt, wenn man aus ihm Schlussfolgerungen für die Gestaltung konkreter politischer Prozesse ziehen möchte. ${ }^{18}$

Diese Überlegungen zeigen, dass normative Aussagen grundsätzlich nicht ohne genauere Kenntnis der Wirklichkeit getroffen werden können, für die aus der normativen Aussage Schlussfolgerungen gezogen werden sollen. Immanuel Kant schrieb einst zur empirischen Rechtslehre: „Eine bloß empirische Rechtslehre ist (wie der hölzerne Kopf in Phädrus' Fabel) ein Kopf, der schön sein mag, nur schade! daß er kein Gehirn hat. ${ }^{\text {“19 }}$ Normative Forschung ohne Empirie scheint dazu das Gegenstück zu sein - ein brillanter Geist ohne Körper, der ihm zum nötigen Bodenkontakt verhilft.

\section{Theorie und Empirie in der Rechtswissenschaft}

Lässt sich diese Erkenntnis der empirischen Bedingtheit normativer Theorien auch auf die Rechtswissenschaft übertragen? Immerhin gibt es zwischen der politischen Theorie und der Philosophie auf der einen sowie der Rechtswissenschaft auf der anderen Seite einen entscheidenden Unterschied - letztere beschäftigt sich mit positiven Normen, die die Grundlage jeder normativen Aussage sein sollen. Die Rechtsordnung kann Festsetzungen treffen, an die der Rechtsan-

15 Jürgen Habermas, Faktizität und Geltung. Beiträge zur Diskurstheorie des Rechts und des demokratischen Rechtsstaats, 1992, S. 349 ff.; Jürgen Habermas, Drei normative Modelle der Demokratie, in: ders., Die Einbeziehung des Anderen. Studien zur politischen Theorie, 1996, 277; Rainer Schmalz-Bruns, Reflexive Demokratie. Die demokratische Transformation moderner Politik, 1995.

16 Habermas, Faktizität (Fn. 15), 369 (Hervorhebung hier).

17 Für einen guten Überblick s. Cass R. Sunstein, Deliberative trouble? Why groups go to extremes, Yale L. J. 110 (2000), 71.

18 Näher dazu Niels Petersen, Demokratie und Grundgesetz - Veränderungen des Demokratieprinzips in Art. 20 Abs. 2 GG angesichts der Herausforderungen moderner Staatlichkeit, JöR 58 (2010), i.E.

I. Kant, Die Metaphysik der Sitten, 1977 [1797], S. 336. 
wender unabhängig von den tatsächlichen Umständen gebunden ist. ${ }^{20}$ Selbst wenn man also hypothetisch - davon ausginge, dass die sozialen und gesellschaftlichen Bedingungen in der Bundesrepublik Deutschland eine Demokratie nicht zuließen, stünde ein Abrücken von der demokratischen Staatsform rechtlich nicht zur Diskussion. Dafür lässt der Wortlaut von Art. 20 I GG keinen Spielraum. In der rechtswissenschaftlichen Diskussion können also normative Modelle nur bedingt mit empirischen Argumenten kritisiert werden. ${ }^{21}$

Selbst in der Rechtswissenschaft sind normative Modelle jedoch nicht vollständig durch das geschriebene Recht determiniert. ${ }^{22}$ Zunächst sind viele rechtliche Begriffe und Konzepte zu einem gewissen Grade unbestimmt und bedürfen der Auslegung. Bleiben wir im klassischen Methodenkanon, dann spielt die Empirie insbesondere bei der teleologischen Auslegung eine zentrale Rolle (dazu a.). Allerdings sind Rechtsprobleme nicht immer allein Probleme der Normbedeutung. Sie können auch Probleme der Auflösung von Normkollisionen sein. Wie Normkollisionen aufzulösen sind, ist teilweise durch mehr oder weniger eindeutige Regeln determiniert. So geht das jüngere dem älteren, das speziellere dem allgemeineren Gesetz vor. Es gibt jedoch auch Fälle, in denen keine abstrakte Rangfolge zwischen Normen besteht. Dies ist vor allem bei der Abwägung konkurrierender Rechtsgüter im Verfassungsrecht der Fall. Auch hier wird oft auf empirische Gesetzmäßigkeiten zurückzugreifen sein (dazu b.).

Schließlich stößt die klassische juristische Auslegung in einigen Fällen an Grenzen. Zum einen kann nicht jede Auslegungsfrage durch den traditionellen Methodenkanon eindeutig beantwortet werden, zum anderen sehen sich Gerichte immer wieder gezwungen, Rechtsfortbildung zu betreiben in Bereichen, in denen die vorhandenen Rechtsnormen für bestimmte Konfliktlagen keine zufriedenstellenden Lösungen bereitstellen. ${ }^{23}$ In diesen Fällen wird oft von einer Normkonkretisierung $^{24}$ gesprochen, die ebenfalls empirischen Bedingungen unterliegt (dazu c.). Im Folgenden sollen diese drei Bereiche näher erläutert und jeweils mit einem Beispiel illustriert werden.

20 Christoph Möllers, Gewaltengliederung - Legitimation und Dogmatik im nationalen und internationalen Rechtsvergleich, 2005, S. 37.

21 Ebd., S. 36 f. und 50.

22 Josef Esser, Vorverständnis und Methodenwahl in der Rechtsfindung, 1970, S. 133-38.

23 Robert Alexy, Theorie der juristischen Argumentation, 1978, S. 17-18. Vgl. auch Matthias Jestaedt, Das mag in der Theorie richtig sein..., 2006, S. 61, der darauf hinweist, dass die Figur der Rechtsfortbildung mit den klassischen Methoden nicht zu fassen sei, sich aber gleichzeitig auch gegen das Konzept der Normkonkretisierung wendet, da dieses kaum Gesetzesbindung gewährleiste.

24 Zum Konzept der Normkonkretisierung s. Ralph Christensen, Was heißt Gesetzesbindung? - Eine rechtslinguistische Untersuchung, 1989, S. 152-58; Friedrich Müller/Ralph Christensen, Juristische Methodik. Band I: Grundlegung für die Arbeitsmethoden der Rechtspraxis, 2009, S. 259-94; Konrad Hesse, Grundzüge des Verfassungsrechts der Bundesrepublik Deutschland, 1995, Rn. 60 ff. 


\section{a. Teleologische Auslegung - Sportwetten als Glücksspiel?}

Die teleologische Auslegung ist die Interpretation einer Norm aufgrund ihres Zwecks. Bei dieser Auslegung greifen normative und empirische Elemente ineinander. ${ }^{25}$ Zunächst muss der Zweck der Norm bestimmt werden, anhand dessen die Norm auszulegen ist. Hierbei handelt es sich vorrangig um eine normative Wertungsfrage. ${ }^{26}$ In einem zweiten Schritt muss dann jedoch bestimmt werden, welche Auslegung diesen Zweck am besten erfüllt - was in erster Linie eine faktische Untersuchung ist. ${ }^{27}$ Dies soll an einem Beispiel aus dem Glücksspielrecht erläutert werden, namentlich der Diskussion, ob es sich bei Sportwetten um Glücksspiele handelt. ${ }^{28}$

Ausgangsnorm des Glücksspielrechts ist $§ 284$ StGB, demzufolge die Veranstaltung von Glücksspielen ohne Genehmigung strafbar ist. Der zentrale Begriff dieser Norm ist der des Glücksspiels. Dieses wird in der Regel von zwei anderen Spieltypen unterschieden: dem Unterhaltungsspiel, in dem es nicht um einen finanziellen Gewinn geht und dem Geschicklichkeitsspiel, bei dem nicht das Glück, sondern die Fähigkeiten des Spielers im Vordergrund stehen. ${ }^{29}$ Bei den Sportwetten steht vor allem die letztere Unterscheidung im Vordergrund: Handelt es sich bei einer Sportwette möglicherweise eher um ein Geschicklichkeits- als um ein Glücksspiel?

Die Abgrenzung ist nicht ganz einfach. Zwar gibt es Extremfälle, die relativ eindeutig zuzuordnen sind: ${ }^{30}$ So ist eine Lotterie ein reines Glücksspiel, dessen Ausgang durch die Fähigkeit des Spielers nicht beeinflusst werden kann; dagegen ist ein Wissensquiz ein Geschicklichkeitsspiel, da dieses allein von den Fähigkeiten des Spielers und nicht von anderen unwägbaren Faktoren abhängt. Dazwischen gibt es jedoch eine Grauzone, in der sowohl Geschicklichkeit als auch Glück eine Rolle spielen und in der vieles unsicher ist.

Für die Abgrenzung kann man dabei mehrere Maßstäbe wählen: Man kann von einem Geschicklichkeitsspiel etwa dann ausgehen, wenn die Fähigkeiten des Spielers einen nachweislichen Einfluss auf dessen Gewinnchancen haben, gute Spieler also regelmäßig besser abschneiden als schlechte. ${ }^{31}$ Alternativ kann man aber auch - wie die wohl überwiegende Meinung - danach fragen, ob durchschnittliche Spieler die Fähigkeit haben, den Spielverlauf durch ihre Geschicklichkeit zu beeinflussen. ${ }^{32}$ Welchen Maßstab man für die Bestimmung des Glücksspielbegriffs wählt,

25 S. etwa Christoph Engel, Rationale Rechtspolitik und ihre Grenzen, JZ 2005, 581, 582, der die teleologische Auslegung als trojanisches Pferd bezeichnet, mit dem Konzepte der Nachbarwissenschaften in die Rechtswissenschaft Eingang finden.

26 Allerdings ist ein Verständnis empirischer Zusammenhänge immer auch Voraussetzung korrekter normativer Wertungen. S. dazu Stefan Magen, Rechtliche und ökonomische Rationalität im Emissionshandelsrecht, in: Emanuel Towfigh et al. (Hg.), Recht und Markt - Wechselbeziehungen zweier Ordnungen, 2009, 9, 18-28, der zeigt, dass die Annahme, dass Zuteilungsregeln im Emissionshandelsrecht Anreizwirkungen haben sollen, auf einem fehlenden ökonomischen Verständnis beruht.

27 Van Aaken (Fn. 6), 91.

28 S. dazu BVerwGE 96, 293 - Sportwetten; BVerwGE 114, 92 - Oddset.

29 Zu diesen Abgrenzungen s. BGHSt 2, 274 (276); 29, 152 (157).

30 S. Andreas Voßkuhle, Glücksspiel zwischen Staat und Markt, VerwArch 87 (1996), 395 (401).

31 So Andreas Glöckner/Emanuel Towfigh, Geschicktes Glücksspiel - Die Sportwette als Grenzfall des Glücksspielrechts, Preprints of the Max Planck Institute for Research on Collective Goods, 2010, i.E.

32 S. nur Herbert Tröndle/Thomas Fischer, Strafgesetzbuch, 54. Aufl. 2007, § 284, Rn. 8; Schönke/SchröderAlbin Eser/Günter Heine, Strafgesetzbuch, 27. Aufl. 2006, § 284, Rn. 5. 
ist letztlich vor allem eine normative Wertungsfrage. ${ }^{33}$ Allein aufgrund des Begriffs des Glücksspiels ist eine sinnvolle Abgrenzung zwischen beiden Spielarten nicht möglich. Vielmehr bietet sich eine teleologische Auslegung an. Geht man davon aus, dass der Zweck des Glückspielverbots in erster Linie die Vermeidung von Spielsucht ist, ${ }^{34}$ dann müsste man den Unterscheidungsmaßstab nehmen, der diesem Ziel besser gerecht wird - oder aber einen dritten Maßstab finden, der möglicherweise noch mehr zur Vermeidung von Spielsucht beiträgt als die beiden dargestellten. ${ }^{35}$

Hierbei handelt es sich jedoch um eine rein empirische Frage. Diese unterscheidet sich dabei vom klassischen Subsumtionsvorgang. Es muss nicht nur einfach entschieden werden, ob es sich bei Sportwetten um Glücksspiele handelt. Vielmehr ist, akzeptiert man die hier gemachten Prämissen, entscheidend, ob diese zur Spielsucht beitragen oder nicht. Hierbei handelt es sich nicht um eine Frage nach Beobachtungen im Einzelfall, sondern nach allgemeinen sozialen GesetzmäBigkeiten, deren Beantwortung letztlich Voraussetzung für die Subsumtion ist.

\section{b. Abwägung im Verfassungsrecht - die Entscheidung des BVerfG zum Rauchverbot}

Bei der Grundrechtsprüfung im Verfassungsrecht spielen empirische Erkenntnisse in erster Linie an zwei Stellen eine zentrale Rolle: zum einen bei der Prüfung der Erforderlichkeit und zum anderen bei der Abwägung im Rahmen der Angemessenheit. Ein Grundrechtseingriff ist immer nur dann erforderlich, wenn es keine milderen, gleich geeigneten Mittel zur Erreichung desselben Ziels gibt. ${ }^{36}$ Sowohl bei der Frage, ob ein Mittel weniger intensiv in ein Grundrecht eingreift, als auch bei der, ob es in gleichem Maße geeignet ist, handelt es sich jeweils um faktische Fragen, die eine beträchtliche Komplexität erreichen können. ${ }^{37}$ Gerade bei komplexen Fragestellungen umgeht das Verfassungsgericht eine nähere Beschäftigung mit der Empirie oft dadurch, dass es dem Gesetzgeber eine Einschätzungsprärogative einräumt. ${ }^{38}$ Diese Einschätzungsprärogative ist sicherlich gerechtfertigt, soweit es um normative Wertungen oder um die Entscheidung geht, wie mit bestehender Unsicherheit im konkreten Fall umzugehen ist. ${ }^{39}$ Ein Verzicht auf faktische Aufklärung selbst in komplizierten Fällen legitimiert sie dagegen nicht.

Bei der Angemessenheit geht es darum, mittels Abwägung einen Ausgleich zwischen dem beeinträchtigten Grundrecht und dem mit der Grundrechtsbeeinträchtigung verfolgten Ziel zu finden. Solche Abwägungen werden im Verfassungsrecht nicht abstrakt vorgenommen. ${ }^{40}$ Vielmehr erfolgt die Auflösung des Konflikts aufgrund der konkreten Umstände des Falles, wobei die

33 Vgl. Andreas Voßkuhle/Christian Bumke, Rechtsfragen der Sportwette, 2002, S. 24.

34 Ebd., 16-17.

35 So auch Glöckner/Towfigh (Fn. 31).

36 BVerfGE 30, 292 (316).

37 S. dazu Christoph Engel, Das legitime Ziel als Element des Übermaßverbots, in: Winfried Brugger/Stephan Kirste/Michael Anderheiden (Hg.), Gemeinwohl in Deutschland, Europa und der Welt, 2002, 103, 113, der von einem Institutionenvergleich spricht.

38 Klaus Meßerschmidt, Gesetzgebungsermessen, 2000, S. 940-41.

39 S. zu letzterem I. Spiecker gen. Döhmann, Staatliche Entscheidungen unter Unsicherheit (Habilitationsschrift), Osnabrück 2007, S. $450 \mathrm{ff}$.

40 Robert Alexy, Theorie der Grundrechte, 1994, S. 78 f. und 100 f. 
Schwere der Beeinträchtigung eine zentrale Rolle spielt. ${ }^{41}$ Wie schwer eine Beeinträchtigung ist, ist jedoch ebenfalls vor allem eine faktische Frage. Auch in diesem Zusammenhang arbeitet das Gericht gerne mit Einschätzungsspielräumen. Auch hier gilt, dass solche bei normativen Wertungen und dem Umgang mit Unsicherheit gerechtfertigt sind, jedoch keine Absolution erteilen, sich nicht mit komplexen empirischen Fragestellungen zu beschäftigen.

Ein Beispiel aus der Rechtsprechung des Bundesverfassungsgerichts soll dies erläutern - die Entscheidung des ersten Senats zum Rauchverbot. ${ }^{42}$ Bei dieser Entscheidung musste ein Ausgleich zwischen zwei konkurrierenden Verfassungsgütern gefunden werden: auf der einen Seite dem Gesundheitsschutz der nicht rauchenden Gäste und Bediensteten einer Lokalität des Gaststättengewerbes und auf der anderen Seite der Berufsfreiheit der Gaststättenbetreiber. Welches der beiden Verfassungsgüter im konkreten Fall den Vorrang hat, hängt insbesondere davon ab, wie stark die jeweilige Beeinträchtigung ist. Hat das Passivrauchen schwere gesundheitliche Folgen, oder handelt es sich lediglich um eine lästige Unannehmlichkeit? Wie groß und wie dauerhaft sind die wirtschaftlichen Auswirkungen des Rauchverbots auf die Gaststättenbetreiber? Die Frage, welchem Rechtsgut konkret der Vorzug zu geben ist, ist letztlich eine normative Frage. Diese normative Wertung wird aber von den faktischen Umständen wesentlich beeinflusst.

In Bezug auf den Gesundheitsschutz prüft das Verfassungsgericht, ob sich die Landesgesetzgeber bei ihrer Entscheidung auf ausreichende tatsächliche Grundlagen gestützt haben. ${ }^{43}$ Dabei stützt es sich im Wesentlichen auf medizinische Sachverständigengutachten. Es stellt zwar fest, dass bei den Auswirkungen des Passivrauchens auf die Gesundheit in Fachkreisen eine gewisse Unsicherheit zu bestehen scheint, gesteht dem Gesetzgeber aber zu, dass er diese Unsicherheit durch die Anwendung des Vorsorgeprinzips löst und sich im Zweifel für den Gesundheitsschutz entscheidet. $^{44}$

Im Rahmen der Abwägung stellt das Gericht fest, dass das Rauchverbot einen schweren Eingriff in die Berufsausübungsfreiheit der Gastwirte darstelle. ${ }^{45}$ Diese Feststellung stützt es im Wesentlichen auf Befunde des statistischen Bundesamtes, denen zufolge die Umsatzrückgänge für die Gastronomie in Bundesländern mit Rauchverbot deutlich stärker waren als in Ländern ohne Rauchverbot. ${ }^{46}$ Weiterhin führt es eine Befragung von Rauchern an, in der diese angeben in $\mathrm{Zu}$ kunft häufiger oder ausschließlich Kneipen aufzusuchen, die vom Rauchverbot ausgenommen werden. $^{47}$

Die konkrete normative Abwägung zwischen den beiden konkurrierenden Grundrechten überlässt das Verfassungsgericht letztlich jedoch den jeweiligen Landesgesetzgebern. ${ }^{48}$ Es gesteht diesen zu, unterschiedliche Konzeptionen für den Ausgleich beider Rechtsgüter zu verfolgen -

41 Hans Dieter Jarass, in: Jarass/Pieroth, Grundgesetz, 10. Aufl. 2009, Art. 20, Rn. 86.

42 BVerfGE 121, 317 - Rauchverbot

43 Ebd., 352.

44 Ebd., $352 \mathrm{f}$.

45 Ebd., 355.

46 Vgl. Pressemitteilung des Statistischen Bundesamts vom 6. Juni 2008 - 207/08.

47 BVerfG (Fn. 42), 363 f.

48 Ebd., $356 \mathrm{f}$. 
dabei sei sowohl ein vollständiges Rauchverbot als auch ein stringentes Rauchverbot mit gewissen Ausnahmen oder ein Verzicht auf ein Rauchverbot möglich. Es behält sich dabei jedoch eine Kohärenzprüfung vor. ${ }^{49}$ Aufgrund dieser Prüfung hält es die angegriffenen Regelungen für verfassungswidrig, da diese die zugrunde gelegte Schutzkonzeption nicht kohärent verfolgt, sondern durch die Art der eingeführten Ausnahmen verwässert habe. ${ }^{50}$

Das Gericht verfolgt im Umgang mit der empirischen Seite der Grundrechtskollision also einen doppelten Ansatz. Zunächst betreibt es selbst empirische Aufklärung, wobei auffällt, dass das Gericht sich für medizinischen Teil auf Sachverständigengutachten stützt, während es die ökonomischen Daten, die Auswirkung des Rauchverbots auf den Umsatz, selbst interpretiert. ${ }^{51}$ Die normative Bewertung dieser sozialen Fakten nimmt es dann aber nur begrenzt vor und gesteht dem Gesetzgeber einen Einschätzungsspielraum zu. Allerdings hat dieser Einschätzungsspielraum Grenzen. Aufgrund der empirischen Feststellung, dass das Rauchverbot einen schweren Eingriff in die Berufsfreiheit bedeute, behält sich das Gericht vor, eine Kohärenzprüfung vorzunehmen, der die geprüften Regelungen zum Opfer fallen. ${ }^{52}$

\section{c. Normkonkretisierung - das Lissabon-Urteil des BVerfG}

Das Konzept der Normkonkretisierung beruht auf der Annahme, dass Normsätze mittels der traditionellen methodischen Instrumente nicht vollständig aus dem positiven Recht deduzierbar ist. ${ }^{53}$ Vielmehr hängt die Konkretisierung in nicht unerheblichem Maße auch vom Hintergrund des Norminterpreten ab. ${ }^{54}$ Beispiele für konkretisierungsbedürftige Rechtsbegriffe findet man vor allem im Verfassungsrecht mit seinen vagen und generellen Konzepten. ${ }^{55}$ Was unter Demokratie oder Menschenwürde zu verstehen ist, wird im Verfassungstext nicht näher erläutert und kann auch aus Systematik oder Entstehungsgeschichte nicht eindeutig hergeleitet werden.

In diesem Kontext ähnelt rechtswissenschaftliche Theoriebildung in hohem Maße der allgemeinen Bildung normativer Theorien in Philosophie oder politischer Theorie, so dass die Ausführungen, die dort zur empirischen Bedingtheit normativer Theorien gemacht wurden, ${ }^{56}$ im Grund-

49 Zu dieser Figur der Folgerichtigkeit s. Lothar Michael, Folgerichtigkeit als Wettbewerbsgleichheit - Zur Verwerfung von Rauchverboten in Gaststätten durch das BVerfG, JZ 2008, 875.

50 Dass man über die Bewertung der Kohärenz unterschiedlicher Ansicht sein kann, zeigt die abweichende Meinung des Richters Masing, BVerfGE 121, $381 \mathrm{ff}$.

51 Vgl. Oliver Lepsius, Sozialwissenschaften im Verfassungsrecht - Amerika als Vorbild?, JZ 2005, 1, 2, demzufolge diese Sichtweise in der Juristerei Tradition hat.

52 S. dazu die Kritik in der abweichenden Meinung des Richters Bryde, BVerfGE 121, 378 (380), der dem Gesetzgeber auch in empirischen Fragen und nicht nur bei deren normativer Bewertung einen Einschätzungsspielraum zugestehen möchte.

53 Müller/Christensen (Fn. 24), S. 260.

$54 \quad$ Hesse (Fn. 24), Rn. 62 f.

55 Ebd., Rn. 60; Andreas von Arnauld, Die Wissenschaft vom Öffentlichen Recht nach einer Öffnung für sozialwissenschaftliche Theorie, in: Andreas Funke/Jörn Lüdemann (Hg.), Öffentliches Recht und Wissenschaftstheorie, 2009, 65, 75. Vgl. auch Jörn Lüdemann, Netzwerke, Öffentliches Recht und Rezeptionstheorie, in: Sigrid Boysen et al. (Hg.), Netzwerke, 2007, 266, 279, der darauf hinweist, dass der Nutzen sozialwissenschaftlicher Erkenntnisse umso größer ist, je abstrakter die rechtliche Norm ist.

S. dazu oben, II 1. 
satz auch auf die Rechtswissenschaft Anwendung finden. ${ }^{57}$ Dies soll im Folgenden an den Ausführungen des Bundesverfassungsgerichts im Lissabon-Urteil ${ }^{58}$ zur Demokratietheorie näher erläutert werden.

Eine der Innovationen des Lissabon-Urteils ist die Identifizierung bestimmter Kompetenzbereiche, die grundsätzlich dem Nationalstaat vorbehalten sind und daher nicht auf supranationale Organisationen wie die Europäische Union übertragen werden können. ${ }^{59}$ Die Argumentation ist dabei ausnahmslos deduktiv, als scheine das Gericht seine Konzeption allein aus dem positiven Normwerk herzuleiten. Danach sei Demokratie ohne öffentliche Meinung nicht funktionsfähig. ${ }^{60}$ Die öffentliche Diskussion von Sachthemen sei jedoch an „,nationalstaatliche, sprachliche, historische und kulturelle Identifikationsmuster angeschlossen“. ${ }^{61}$ Daraus zieht das Verfassungsgericht die normative Schlussfolgerung, dass ein strukturelles Demokratiedefizit vorläge, wenn die ausgemachten Kernkompetenzen des Staates auf Unionsebene ausgeübt würden. ${ }^{62}$

Die beiden Prämissen, aus denen die Schlussfolgerung gezogen wird, sind jeweils empirische Annahmen. Für die erste, dass Demokratie ohne öffentliche Meinung nicht funktionsfähig sei, ist im Urteil immerhin einer plausible Begründung angedeutet: Eine freie und kritische öffentliche Meinung sei notwendig, um die politische Leistung von Regierung und Parlamentsmehrheit zu bewerten und diese für ihre Entscheidungen verantwortlich zu machen. ${ }^{63}$ Problematischer ist aber die zweite empirische Prämisse - die Beobachtung, dass sich die öffentliche Meinung in erster Linie nationalstaatlich konstituiere, sowie die unausgesprochene Annahme, dass eine - wie auch immer zu definierende - gesamteuropäische öffentliche Meinung notwendig sei, um auf europäischer Ebene politische Entscheidungen den Verantwortungsträgern zurechnen zu können. ${ }^{64}$ Im Urteil findet sich dazu eine bloße Behauptung (,,ist nicht zu übersehen ${ }^{\text {“65 }}$ ). Eine Begründung oder gar empirische Evidenz fehlen vollständig. Das Gericht scheint davon auszugehen, dass diese empirische Annahme intuitiv unmittelbar einsichtig ist, riskiert damit aber, dass die Begründung nur diejenigen Leser des Urteils überzeugt, die an einen solchen Zusammenhang tatsächlich glauben.

Ähnlich Lepsius (Fn. 51), 1.

BVerfG, Urt. v. 30. Juni 2009, Az.: 2 BvE 2/08 u.a., NJW 2009, 2267 = JZ 2009, 890 - Lissabon.

59 S. dazu Frank Schorkopf, Die Europäische Union im Lot - Karlsruhes Richterspruch zum Vertrag von Lissabon, EuZW 20 (2009), 718, 719; Daniel Halberstam/Christoph Möllers, The German Constitutional Court says "Ja zu Deutschland!", German L.J. 10 (2009), 1241, 1250.

60 BVerfG (Fn. 58), Rn. 250.

61 Ebd., Rn. 251. Das Argument geht zurück auf Dieter Grimm, Braucht Europa eine Verfassung?, JZ 1995, 581, 588. Zust. Klaus Ferdinand Gärditz/Christian Hillgruber, Volkssouveränität und Demokratie ernst genommen - Zum Lissabon-Urteil des BVerfG, JZ 2009, 872, 880.

62 BVerfG (Fn. 58), Rn. 264.

63 Ebd., Rn. 268.

64 Schließlich müsste in einem weiteren Schritt noch ausgeführt werden, warum sich aus der Erforderlichkeit einer einheitlichen öffentlichen Meinung gerade die fünf vom Gericht identifizierten Kompetenzbereiche als notwendige Staatsaufgaben herleiten lassen. Auch eine Theorie hierzu lässt das Gericht aber vermissen, s. Halberstam/Möllers (Fn. 59), 1250; Jo Eric Khushal Murkens, Identity Trumps Integration - The Lisbon Treaty in the German Federal Constitutional Court, Der Staat 48 (2009), 517, 523. 
Denn es gibt durchaus Anhaltspunkte, Zweifel an den empirischen Prämissen anzumelden. ${ }^{66} \mathrm{Ob}$ man eine europäische Öffentlichkeit feststellen kann, hängt sicherlich davon ab, wie man diese Öffentlichkeit definiert. Ist es die Beschäftigung mit gemeinsamen Themen, die Konsumtion derselben Medien oder möglicherweise noch ein dritter Faktor? Wie stark müssen Überschneidungen in dieser Hinsicht sein? Und vor allem: Inwieweit ist das notwendig, um eine Verantwortlichkeit europäischer Politik zu gewährleisten? Dem Urteil fehlt in dieser Hinsicht bereits die analytische Klarheit, von der empirischen Evidenz ganz zu schweigen. Insofern erscheint das Vorgehen des Gerichts, empirische Fragen durch normative Setzungen einfach zu umgehen, äußerst fragwürdig. ${ }^{67}$

\section{d. Fazit}

Die vorhergehenden Überlegungen zeigen, dass normative Aussagen auch in der Rechtswissenschaft durchaus auf empirischen Prämissen beruhen. Zwar ist der Einfluss schwächer als in der Philosophie oder der politischen Theorie, da die Juristerei immer positive Normen als Ausgangspunkt nehmen muss. Allerdings determinieren diese eine juristische Entscheidung selten in dem Maße, dass faktische Erwägungen vollständig an Relevanz verlören. Bedeutung hat der Rückgriff auf die Empirie vor allem in drei Fallgruppen - bei der teleologischen Auslegung, bei der Verhältnismäßigkeitsprüfung im Verfassungsrecht, sowie bei der Normkonkretisierung. Bei der teleologischen Auslegung und bei der Verhältnismäßigkeitsprüfung kann man den empirischen Bezug dabei mit einigem Wohlwollen noch als Erweiterung des Kanons ansehen. Der Unterschied zur klassischen Subsumtion besteht vor allem darin, dass die empirischen Untersuchungen auf allgemeine soziale Gesetze statt auf den Einzelfall bezogen sind und gleichzeitig Voraussetzung und nicht Teil der Subsumtion sind.

Das klassische Modell wird aber bei der Normkonkretisierung verlassen. Dies ist nicht weiter überraschend, da diese Art der Interpretation von der Annahme abweicht, dass das Recht vollständig aus geschriebenen Normen deduziert werden könne. In diesem Zusammenhang stellen sich denn auch ähnliche Probleme wie im Bereich humanistischer Normwissenschaften wie der Philosophie oder der politischen Theorie. Normative Aussagen sind von bestimmten empirischen Voraussetzungen abhängig. Somit kommt anspruchsvolle juristische Theoriebildung in Wissenschaft und Rechtsprechung ebenfalls nicht ohne Rückgriff auf empirische Erkenntnisse aus.

66 Für eine prononcierte Kritik an vergleichbaren Annahmen im Maastricht-Urteil s. Joseph H.H. Weiler, Does Europe Need a Constitution? Demos, Telos and the German Maastricht Decision, ELJ 1 (1995), 219, 224-31.

67 Vgl. in anderem Zusammenhang die ähnliche Kritik von Christoph Möllers, Braucht das öffentliche Recht einen neuen Methoden- und Richtungsstreit?, VerwArch 90 (1999), 187, 204. 


\section{Empirische Studien und normative Werturteile}

Der vorherige Abschnitt hat gezeigt, dass auch normative Wissenschaften wie die Rechtswissenschaft nicht um die Beschäftigung mit empirischer Forschung herumkommen. Wie aber sollte diese Beschäftigung aussehen? Eine Möglichkeit, zu der die Rechtsprechung des Bundesverfassungsgerichts oft zu tendieren scheint, ist es, sozialwissenschaftliche Aussagen implizit oder explizit selbst zu treffen. ${ }^{68}$ Dieser Weg ist aber nicht unproblematisch. Es gibt in den Sozialwissenschaften ein Instrumentarium, das Regeln aufstellt, inwieweit aus faktischen Beobachtungen auf allgemeine Gesetzmäßigkeiten geschlossen werden kann. Missachtet man diese Regeln, besteht die Gefahr, dass von zufälligen Koinzidenzen fälschlicherweise auf das Bestehen eines kausalen Zusammenhangs geschlossen wird (dazu 1.).

Eine oft diskutierte Alternative zur Selbstbewertung sozialer Tatsachen ist eine Arbeitsteilung mit Sozialwissenschaftlern. ${ }^{69}$ Juristen beschäftigen sich in erster Linie mit normativen Fragestellungen und informieren sich über die Ergebnisse empirischer Forschung aus anderen Disziplinen. Für die Praxis folgt daraus der Rückgriff auf Sachverständigengutachten, für die Wissenschaft die Rezeption sozialwissenschaftlicher Forschung. Empirische Studien werden gewissermaßen als black box begriffen, deren Ergebnisse von Juristen nutzbar gemacht werden, ohne die genauen Voraussetzungen der Studie zu kennen, so wie man einen Computer als Arbeitsmittel benutzt, ohne sich mit den technischen Einzelheiten auskennen zu müssen.

Ein solcher Ansatz birgt jedoch Gefahren. Selbst wenn man davon ausgeht, dass eine Studie den methodischen Standards ihrer Wissenschaft entspricht, kann sie nicht ohne weiteres in den juristischen Kontext übertragen werden. Vielmehr muss man sich der Voraussetzungen der Studie immer bewusst sein. Insbesondere enthalten auch viele empirische Studien implizite normative Wertungen. Möchte man eine empirische Studie für die Rechtswissenschaft fruchtbar machen, muss man sich dieser normativen Wertungen bewusst sein, um die Grenzen der Übertragbarkeit in den juristischen Kontext beurteilen zu können (dazu 2.).

\section{Empirie und Validität}

Wenn wir bestimmte soziale Phänomene beobachten, können wir daraus noch nicht notwendigerweise Schlüsse über kausale Zusammenhänge ziehen. Nur weil X und Y gleichzeitig auftreten, heißt dies noch nicht, dass $\mathrm{Y}$ auch durch $\mathrm{X}$ bewirkt worden ist. Vielmehr gibt es hier in dreifacher Hinsicht Probleme, die sich unter dem Stichwort der Validität zusammenfassen lassen: ${ }^{70}$ zunächst kann das gleichzeitige Auftreten beider Faktoren auf bloßem Zufall beruhen (statistische Validität). Dass wir Y beobachten, liegt möglicherweise an einem dritten Faktor W, den wir

68 S. dazu Lepsius (Fn. 51), 4.

69 Für eine solche plädieren etwa Jörn Lüdemann, Rechtsetzung und Interdisziplinarität in der Verwaltungsrechtswissenschaft, in: Andreas Funke/Jörn Lüdemann (Hg.), Öffentliches Recht und Wissenschaftstheorie, 2009, 125, 131-37 und mit Einschränkung auch Lepsius (Fn. 51), 12.

70 S. dazu ausführlich Thomas D. Cook/Donald T. Campbell, Quasi-Experimentation. Design \& Analysis Issues for Field Settings, 1979, S. 37-94. 
jedoch in unserer Studie nicht berücksichtigt haben. Nur weil wir kurz vor einem Unfall eine schwarze Katze gesehen haben, können wir noch nicht automatisch davon ausgehen, dass das Erspähen eines solchen Tieres immer zu einem Unfall führen muss.

Weiterhin ist es möglich, dass wir zwar einen statistisch signifikanten Zusammenhang zwischen $\mathrm{X}$ und $\mathrm{Y}$ beobachten, dieser aber auf einen dritten Faktor V zurückzuführen ist (interne Validität), der sowohl $\mathrm{X}$ als auch $\mathrm{Y}$ verursacht. So kann man einen statistisch signifikanten Zusammenhang zwischen dem Rückgang der Storchpopulation in Deutschland und dem Geburtenrückgang ausmachen. ${ }^{71}$ Ist es also doch der Storch, der uns die Kinder bringt? Die Freude, ein kontraintuitives soziales Phänomen entdeckt zu haben, währt jedoch nur kurz, wenn wir uns klar machen, dass beide Beobachtungen möglicherweise auf ein drittes Phänomen zurückzuführen sind - die Industrialisierung.

Schließlich ist es möglich, dass wir zwar einen kausalen Zusammenhang zwischen zwei Phänomenen beobachten können, dieser aber möglicherweise von bestimmten Nebenbedingungen abhängt, und daher über den Kontext hinaus, in dem wir ihn beobachtet haben, nicht generalisierbar ist (externe Validität). Zum einen können die Ergebnisse einer Studie nur für die Population generalisiert werden, aus der sie gezogen worden ist. ${ }^{72}$ Wird unsere Stichprobe also aus deutschen Studenten rekrutiert, dann können wir daraus noch keine Folgerungen für chinesische Bauarbeiter oder Unternehmensberater ziehen. ${ }^{73}$ Schließlich ist es wichtig, immer die Bedingungen zu beachten, unter denen eine bestimmte Studie durchgeführt worden ist. Herrschen in dem Kontext, in den man die Ergebnisse übertragen möchte andere Umstände, ist eine Übertragbarkeit nicht ohne weiteres möglich. So bedeutet, dass in bestimmten Experimenten herausgefunden wurde, dass Deliberation unter bestimmten Umständen zur Polarisierung der Diskussionsteilnehmer führt, ${ }^{74}$ nicht, dass Deliberation per se zu einer Polarisierung führt.

Welche Bedeutung hat das für den juristischen Kontext? Um dies zu verdeutlichen, kehren wir noch einmal zum Urteil des Bundesverfassungsgerichts zum Rauchverbot zurück. ${ }^{75}$ Die sozialwissenschaftliche Frage, die das Gericht zu beantworten hatte, war, ob das Rauchverbot ökonomische Auswirkungen auf den Gastronomiebetrieb hat. Dabei stützt sich das Gericht im Wesentlichen auf eine Pressemitteilung des Statistischen Bundesamtes. ${ }^{76}$ Bei seiner Begründung, warum das Rauchverbot einen schweren Eingriff in die Berufsfreiheit darstelle, greift es auf die dort genannten Zahlen zurück: weil der Umsatzrückgang bei den Bundesländern mit Rauchverbot stärker sei als bei denen ohne Rauchverbot (-9,8\% gegenüber -6,8\% im 3. Quartal 2007 und 14,1\% gegenüber -8,8\% im 4. Quartal 2007), könne festgestellt werden, dass das Rauchverbot wirtschaftliche Auswirkungen habe.

71 Amüsant in diesem Zusammenhang Thomas Höfer/Hildegard Przyrembel/Silvia Verleger, New evidence for the Theory of the Stork, Paediatric and Perinatal Epidemiology 18 (2004), 88.

$72 \quad$ Leslie Kish, Statistical Design For Research, 1987, S. 9.

73 Vgl. dazu auch von Arnauld (Fn. 55), 92-93, der vor den Gefahren einer Dekontextualisierung am Beispiel von Assmanns Theorie des kulturellen Gedächtnisses warnt.

74 Johannes A. Zuber/Helmut W. Crott/Joachim Werner, Choice Shift and Group Polarization: An Analysis of the Status of Arguments and Social Decision Schemes, J. Pers. \& Soc. Psych. 62 (1992), 50.

75 S.o., Fn. 42.

76 BVerfG (Fn. 42), 355 und 364. 
Allein das Gegenüberstellen dieser Zahlen reicht allerdings nicht aus, um darauf schließen zu können, dass das Rauchverbot einen Einfluss auf die Umsatzentwicklung gehabt hat. Der Umsatz der getränkegeprägten Gastronomie wird durch viele Faktoren beeinflusst - die allgemeine Wirtschaftslage, die soziale und demographische Struktur des Einzugsgebietes oder das Wetter. Das Rauchverbot ist dabei nur ein potentieller Faktor unter mehreren. Das Verfassungsgericht geht mit seiner Gegenüberstellung implizit davon aus, dass diese Faktoren abgesehen vom Rauchverbot in allen Bundesländern exakt gleich waren. Das ist aber mehr als unwahrscheinlich.

Nehmen wir ein konkretes Beispiel, um die Argumentation intuitiv zu verdeutlichen. Dass das Rauchverbot einen Einfluss auf die Umsatzzahlen zu haben scheint, erscheint uns intuitiv plausibel, wenn wir nur den Durchschnitt betrachten. Wir müssen uns allerdings vor Augen führen, dass es sich nur um den Durchschnitt handelt, wir gleichzeitig aber keine Aussagen über die Varianz haben. Diese ist in unserem Fall aber beträchtlich. Dies zeigt die Stichprobe in der folgenden Tabelle, die auf einer Auskunft der entsprechenden Statistischen Landesämter beruht: ${ }^{77}$

\begin{tabular}{l|cc|cc} 
& \multicolumn{2}{|c|}{ 3. Quartal 2007 } & \multicolumn{2}{c}{$\begin{array}{c}\text { Q. Quartal 2007 } \\
\text { Bundesland }\end{array}$} \\
Rauchverbot & kein Rauchverbot & Rauchverbot & kein Rauchverbot \\
\hline Bayern & & $-11,9 \%$ & & $-7,0 \%$ \\
Mecklenburg & & $-21,7 \%$ & $-2,6 \%$ \\
Nordrhein-Westfalen & & $-3,9 \%$ & & $-8,7 \%$ \\
Schleswig-Holstein & & $-2,8 \%$ & & $-2,5 \%$ \\
Thüringen & & $-16,1 \%$ & & $-14,4 \%$ \\
Hessen & & $-14,5 \%$ & & \\
Baden-Württemberg & & & $-9,5 \%$ & \\
Niedersachsen & $-1,4 \%$ & $-6,7 \%$ & $-8,8 \%$ \\
\hline Pressemitteilung & $-8,6 \%$ & $-14,9 \%$ & \\
\hline
\end{tabular}

Betrachten wir diese Daten für die einzelnen Bundesländer, sticht zweierlei ins Auge. Zum einen stimmen die Daten der statistischen Landesämter der Bundesländer mit Rauchverbot nicht mit denen des Statistischen Bundesamtes überein. Im dritten Quartal 2007 können die Bundesländer Baden-Württemberg und Niedersachsen als einzige Länder mit Rauchverbot ebenso wenig auf einen Umsatzrückgang von 9,8\% kommen, wie diese beiden Länder zusammen mit Hessen im vierten Quartal 2007 einen durchschnittlichen Rückgang von 14,1\% verzeichnen können. Diese Differenz ist wohl darauf zurückzuführen, dass die Kategorie der getränkegeprägten Gastronomie beim Bundesamt etwas anders gefasst wird als bei den Landesämtern. ${ }^{79}$ Kleine Änderungen in der Kategorisierung können also schon zu erheblichen Abweichungen im Ergebnis führen. ${ }^{80}$

77 Quellen: Bayern: Daten von der Webseite des Statistischen Landesamtes, http://www.statistik.bayern.de; Mecklenburg-Vorpommern: Auskunft des Statistischen Landesamtes per Email vom 13.1.2010; NordrheinWestfalen: Auskunft des Statistischen Landesamtes per Email vom 6.1.2010; Schleswig-Holstein: Daten von der Webseite des Statistischen Landesamtes für Hamburg und Schleswig-Holstein, http://www.statistiknord.de; Thüringen: Daten von der Webseite des Statistischen Landesamtes, http://www.tls.thueringen.de; Hessen: Auskunft des Statistischen Landesamtes Hessen per Email vom 6.1.2010; Baden-Württemberg: Auskunft des Statistischen Landesamtes per Email vom 7.1.2010; Niedersachsen: Auskunft des Statistischen Landesamtes Niedersachsen per Email vom 17.3.2010.

78 In Hessen wurde das Rauchverbot erst im vierten Quartal 2007 eingeführt.

79 So jedenfalls eine entsprechende Auskunft des Statistischen Bundesamtes per Email vom 21.1.2010.

80 S. dazu noch näher die Ausführungen zu den normativen Wertungen in der Empirie, III 2. 
Zum anderen aber sehen wir, dass zwischen den einzelnen Bundesländern eine erhebliche Varianz besteht. Der Unterschied zwischen den beiden extremen Länder unserer Stichprobe beträgt im dritten Quartal mehr als 20 Prozentpunkte, im vierten Quartal immerhin noch 12,5 Prozentpunkte. Weiterhin ist es nicht so, dass die Umsatzentwicklung in den Ländern mit Rauchverbot durchweg schlechter ausfällt als in den Ländern ohne Rauchverbot. Insofern kann allein aus der Beobachtung eines Unterschieds in der durchschnittlichen Umsatzentwicklung noch nicht auf eine allgemeine Gesetzmäßigkeit geschlossen werden. Diese Beobachtung kann auch auf Faktoren beruhen, die mit der Einführung des Rauchverbots nichts zu tun haben. Ob die Umsatzentwicklung in Niedersachsen oder Baden-Württemberg ohne das Rauchverbot schlechter ausgefallen wäre, das Rauchverbot für diese also kausal war, kann aufgrund dieser Zahlen jedenfalls nicht beurteilt werden. Der Feststellung des Gerichts fehlt damit möglicherweise schon die statistische Validität. $^{81}$

Doch selbst wenn wir davon ausgehen, dass das Rauchverbot einen statistisch signifikanten Effekt auf die Umsatzentwicklung hat, ist zweifelhaft, ob allein der Umsatzrückgang innerhalb des ersten Quartals nach Einführung des Rauchverbots für die Annahme einer schwerwiegenden Beeinträchtigung der Berufsfreiheit ausreicht. Es ist durchaus denkbar und plausibel, dass nach einer gewissen Zeit ein Gewöhnungseffekt eintritt und das Umsatzniveau auf das vorherige Maß zurückkehrt. Es besteht also ein Problem der externen Validität: Eventuell ist der Umsatzrückgang nur der zeitlichen Nähe zur Einführung des Verbots geschuldet, lässt sich aber langfristig nicht beobachten. ${ }^{82} \mathrm{Ob}$ eine Rückkehr zum Ausgangsniveau plausibel ist, ließe sich etwa feststellen, indem man die Entwicklung in anderen Staaten betrachtet, die bereits früher als die deutschen Bundesländer ein Rauchverbot eingeführt haben.

Diese kurzen Ausführungen zeigen, dass sich Juristen ohne nähere Kenntnis empirischer Methodik nicht die empirische Deutungshoheit für soziale Fakten anmaßen sollten. Vielmehr sind in den Sozialwissenschaften Methoden und Regeln entwickelt worden, die Aufschluss darüber geben, inwieweit aus faktischen Beobachtungen auf das Bestehen allgemeiner Gesetzmäßigkeiten gefolgert werden kann. Blendet man dies in der juristischen Argumentation aus, stützt man seine Entscheidungen möglicherweise auf Befunde ohne ausreichende Validität und liegt damit auch in seinen normativen Wertungen schnell falsch. ${ }^{83}$

81 Zutreffend ist in diesem Zusammenhang die Warnung von Christoph Engel, Verhaltenswissenschaftliche Analyse: eine Gebrauchsanweisung für Juristen, in: Christoph Engel/Markus Englerth/Jörn Lüdemann/Indra Spiecker gen. Döhmann (Hg.), Recht und Verhalten, 2007, 363, 367, dass man Ergebnissen ohne Angaben über die statistische Validität auch als Jurist nicht trauen sollte.

82 Natürlich kann man diesem empirischen Argument normativ entgegenhalten, dass ein bereits kurzfristiger Umsatzrückgang schon eine schwerwiegende Beeinträchtigung der Berufsfreiheit darstellt. Allerdings wird es wohl zumindest für das Gewicht der Beeinträchtigung in der Abwägung darauf ankommen, wie groß der Umsatzrückgang ist und wie lange dieser andauert.

83 Daher zu recht auch die Warnung von Andreas Voßkuhle, Das Konzept des rationalen Staates, in: Gunnar Folke Schuppert/Andreas Voßkuhle (Hg.), Governance von und durch Wissen, 2008, 13, 24 vor einem blinden Zahlenfetischismus. 


\section{Empirie und normative Wertungen}

Das bedeutet jedoch nicht, dass der Jurist die Deutung der Wirklichkeit ohne Weiteres dem Sozialwissenschaftler überlassen könnte. Vielmehr gibt es auch in sozialwissenschaftlichen Studien oft normative Wertungen, derer man sich bewusst sein sollte. ${ }^{84}$ Empirische sozialwissenschaftliche Forschung analysiert im Wesentlichen das Bestehen von Kausalzusammenhängen zwischen zwei oder mehr sozialen Faktoren. ${ }^{85}$ Um einen solchen Zusammenhang untersuchen zu können, müssen die für die Studie relevanten Konzepte (Variablen) gemessen werden. Eine solche Messung setzt dabei zwei Schritte voraus: ${ }^{86}$ Zum einen müssen die verwendeten Konzepte definiert, zum anderen müssen sie operationalisiert werden.

Die Definition dient dazu, die entscheidenden Charakteristika eines abstrakten Konzepts herauszustellen. Möchten wir etwa die Auswirkungen der wirtschaftlichen Leistungsfähigkeit eines Staates auf die Stabilität von dessen Demokratie untersuchen, müssen wir zunächst definieren, was unter Demokratie und wirtschaftlicher Leistungsfähigkeit verstanden werden soll. Ist für die Annahme einer Demokratie das Abhalten regelmäßiger Wahlen schon ausreichend? Oder verlangen wir zusätzlich die Achtung bestimmter Grundrechte, wie etwa der Meinungs- und der Pressefreiheit? Allein die Definition reicht zur Messung einer Variable aber noch nicht aus. Vielmehr muss diese in einem zweiten Schritt operationalsiert werden, d.h. es müssen Indikatoren für die Messung gefunden werden. Wenn wir also in unserem Beispiel davon ausgehen, dass die Gewährleistung von Meinungs- und Pressefreiheit ebenfalls entscheidende Charakteristika einer Demokratie sind, müssten wir Indikatoren finden, um diese zu messen.

Vor allem im ersten Schritt, der Definition, kommen dabei normative Wertungen ins Spiel, die das Ergebnis der Studie entscheidend beeinflussen können. Ein Beispiel soll dies verdeutlichen. Nehmen wir an, dass wir vor dem Hintergrund des Sozialstaatsprinzips oder der Menschenwürde - hypothetisch - daran interessiert sind, wie sich eine bestimmte sozialpolitischer Maßnahmen auf das Armutsniveau auswirkt. ${ }^{87}$ Für eine solche Studie müssen wir das Armutsniveau in der Bundesrepublik Deutschland zunächst messen. Konzeptionell haben wir dabei zumindest drei Möglichkeiten. Wir können Armut absolut, relativ oder subjektiv messen.

Ein absoluter Indikator würde ein bestimmtes monatliches Pro-Kopf-Einkommen als Schwelle festlegen, unterhalb derer eine Person oder eine Familie als arm qualifiziert würde. Diese Schwelle könnte sich etwa an dem Preis eines bestimmten statistischen Warenkorbes orientieren - eine Konzeption, die beispielsweise der Bemessung der Regelsätze bei Sozialleistungen

84 S. dazu bereits Engel (Fn. 81), 387-91; von Arnauld (Fn. 55), 90-91.

85 Dies ist eine bewusst etwas verkürzte Darstellung, da es in den Sozialwissenschaften auch eine wichtige Tendenz gibt, die sich eher mit der Interpretation von Bedeutung als mit dem Bestehen von Kausalität beschäftigt, s. dazu etwa Taylor (Fn. 7) ; Clifford Geertz, Thick Description: Toward an Interpretive Theory of Culture, in: ders., The Interpretation of Cultures, 1973, 3; David Dessler/John Owen, Constructivism and the Problem of Explanation, Perspectives on Politics 3 (2005), 597.

86 Royce A. Singleton/Bruce C. Straits, Approaches to Social Research, 5. Aufl. 2010, S. 115-20.

87 Vgl. etwa BVerfG, Urt. v. 9. Feb. 2010 (1 BvL 1/09 u.a.) - Hartz IV, auch wenn die dem Urteil zugrunde liegende Fragestellung eine etwas andere ist, als die hier behandelte. 
zugrunde liegt. ${ }^{88}$ Relative Armut wird demgegenüber gemessen, indem man die Armutsschwelle bei einem bestimmten Prozentsatz des Medianeinkommens festlegt. So gilt nach dem Armutsbericht der Bundesregierung als arm, wer über weniger als 60\% des Medianeinkommens verfügt. ${ }^{89}$ Relative Armut steigt damit in der Regel bei einer größeren Einkommensvarianz, ist also ein Indikator für die Einkommensverteilung, sagt aber nicht zwingend etwas darüber aus, was Menschen, die als arm qualifiziert werden, sich tatsächlich leisten können.

Schließlich ist es denkbar, Armut subjektiv zu bestimmen - etwa mittels eines Fragebogens, in dem die Probanden gefragt werden, ob sie sich in den letzten Monaten bestimmte als notwendig erachtete Ausgaben, beispielsweise die Zahlung der Miete, leisten konnten. Von der absoluten Armutsgrenze unterscheidet sich dieser subjektive Maßstab dadurch, dass Menschen möglicherweise das nötige Geld haben, die Ausgaben aber aufgrund anderer Dispositionsentscheidungen nicht tätigen können. In den subjektiven Armutsmaßstab fließt damit nicht nur das zur Verfügung stehende Geld ein, sondern auch die Fähigkeit von Menschen, mit Geld umzugehen.

Welchen der drei Maßstäbe man einer Studie zugrunde legt, ist eine Entscheidung des jeweiligen Forschers mit normativen Implikationen. Wenn die Fähigkeit mit Geld umzugehen, eine Rolle spielen soll, dann entscheiden wir uns für den subjektiven Maßstab. Wenn es dagegen allein um die materielle Ausgangslage geht, wird einer der objektiven Standards angemessener sein. Bei Fragen der Verteilungsgerechtigkeit mag der relative Standard der bessere Indikator sein. Geht es uns dagegen darum, dass bestimmte Grundbedürfnisse erfüllt werden, ist der objektive Standard wohl aussagekräftiger. Entscheidend für die juristische Entscheidung ist es, diese normativen Implikationen zu beachten. Eine Studie, die Armut misst, ist für die juristische Fragestellungen möglicherweise unpassend, weil ihre normativen Wertungen nicht mit den Wertungen übereinstimmen, die uns das Recht vorgibt.

Ein weiteres Beispiel ist in diesem Zusammenhang die oben bereits angesprochene Glücksspieldefinition in $\S 284$ StGB. $^{90}$ Bei der Unterscheidung zwischen Glücks- und Geschicklichkeitsspielen wird in der Literatur für die Annahme eines Geschicklichkeitsspiels auf unterschiedliche Charakteristika abgestellt. In der empirischen Literatur wird vor allem versucht, das Geschicklichkeitselement danach zu bestimmen, ob Spieler je nach Erfahrung und Talent unterschiedlich abschneiden, bessere Spieler also in der Regel auch tatsächlich besser sind als schlechtere Spieler. ${ }^{91}$ Diese Definition unterscheidet sich von derjenigen, die sich überwiegend in den juristischen Kommentaren zu diesem Thema findet. Letzteren zufolge kommt es darauf an, ob der Durchschnittsspieler das Spiel aufgrund seiner kognitiven Fähigkeiten beeinflussen kann. Danach wäre ein schweres Spiel, bei dem es zwar einige wenige Spieler gibt, die aufgrund ihres Wissens oder ihrer kognitiven Fähigkeiten in der Lage sind, das Spiel zu gewinnen, dann ein

88 S. Verordnung zur Durchführung des $§ 28$ des Zwölften Buches Sozialgesetzbuch v. 3. Juni 2004, BGBl. I S. 1067.

89 Bundesministerium für Arbeit und Soziales (Hg.), Lebenslagen in Deutschland - der 3. Armuts- und Reichtumsbericht der Bundesregierung, S. IX-X.

90 S.o., II 2 a.

91 Jan-Philipp Rock/Ingo Fiedler, Die Empirie des Online-Pokers - Bestimmung des Geschicklichkeitsanteils anhand der kritischen Wiederholungshäufigkeit, ZfWG 3 (2008), 412, 416-18; Glöckner/Towfigh (Fn. 31). 
Glücksspiel, wenn der durchschnittliche Spieler die zum Spielgewinn notwendigen Fähigkeiten nicht besitzt. ${ }^{92}$

Eine empirische Studie, die unter der ersten Definition zu dem Ergebnis kommt, dass der Ausgang eines bestimmten Spiels auf Geschicklichkeit beruht, weil es bessere und schlechtere Spieler gibt, sagt noch nichts darüber aus, ob dasselbe Spiel auch unter der zweiten Definition als Glücks- oder als Geschicklichkeitsspiel einzustufen ist. Dennoch kann auch eine solche Definition den juristischen Diskurs befruchten. Denn die Definition des Glücksspiels ist nirgendwo in Stein gemeißelt - insbesondere ist sie nicht durch das geschriebene Recht determiniert. Insofern kann eine empirische Studie eine Diskussion anstoßen, ob eine bestimmte normative Definition sinnvoll oder praktisch ist. Die Beschäftigung mit der Sozialwissenschaft zeigt auf, dass es für bestimmte Probleme oder Kategorisierungen möglicherweise andere Lösungen oder Wege gibt, die vorher nicht in Erwägung gezogen worden sind.

Ob eine Definition tatsächlich angepasst werden soll, ist letztlich eine normative Frage. Diese wird zum einen von normativen Erwägungen, wie etwa dem mit der Norm verfolgten Zweck abhängen, kann aber durchaus auch auf Praktikabilitätserwägungen beruhen. Eine Definition mag theoretisch noch so elegant sein, wenn die Unterscheidung in der Praxis nicht feststellbar oder durchführbar ist. Im konkreten Fall der Glücksspiele spricht viel dafür, die traditionelle Definition der Literatur aufzugeben. ${ }^{93}$ Stellt man auf den durchschnittlichen Spieler ab, so orientiert sich die Unterscheidung eher daran, ob ein Spiel schwer oder leicht ist, als daran, ob es mit Geschicklichkeit zu tun hat. Zudem bekommt man Probleme bei Spielen, bei denen der Gewinn eines Spielers notwendig mit dem Verlust eines anderen einhergeht, wie etwa beim Schach oder beim Poker. Hier gewinnt der durchschnittliche Spieler per definitionem in genau der Hälfte der Fälle, so dass uns die klassische Definition bei der Unterscheidung keinen Schritt weiterhilft.

\section{Fazit}

In der Theorie sind Normativität und Empirie durch eine feine Linie voneinander getrennt. Es handelt sich um zwei Welten: die Welt des Sollens auf der einen und die des Seins auf der anderen Seite. In der Praxis sind beide aber oft miteinander vermischt. Normative Aussagen beruhen immer auf empirischen Prämissen und empirische Aussagen haben ebenso oft normative Voraussetzungen. In der Rechtswissenschaft wird dieses Ineinandergreifen von Normativität und Empirie oft ignoriert. Entweder versucht man die Empirie durch scheinbare normative Setzungen oder auf Intuition anspielende Behauptungen zu umgehen; ${ }^{94}$ oder man greift auf externe Sachverständige zurück, ohne die empirischen und normativen Voraussetzungen ihrer Studien ausreichend zu verstehen.

92 So etwa die juristische Literatur zum Hütchenrücken, das dann als Glücksspiel angesehen wird, wenn der durchschnittliche Spieler nicht die kognitiven Fähigkeiten hat, um zu verfolgen, unter welchem Hut die Münze liegt. S. dazu Eser/Heine (Fn. 32), Rn. 5.

93 Glöckner/Towfigh (Fn. 31).

94 Lüdemann (Fn. 69), 124. 
Vor einigen Jahren hatte Sofia Coppola mit ihrem Film Lost in Translation einen großen Erfolg, in dem sie uns die Schwierigkeiten des Dolmetschens vor Augen führt, des Übersetzens von einer Sprache in eine andere. Es ist nicht damit getan, die jeweiligen Vokabeln und deren Wörterbuchbedeutung zu kennen. Vielmehr erfordert eine gute Übersetzung immer auch eine Kenntnis der Kulturen der beteiligten Sprachen. Ansonsten kann es schnell zu Missverständnissen kommen - wir alle kennen Beispiele von Hinweisschildern oder Gebrauchsanleitungen, die mittels eines Programms mechanisch übersetzt worden sind, und deren Ergebnis doch nur Wortsalat ist. Ähnlich ist es mit dem Verhältnis der Rechts- und der Sozialwissenschaften. Auch hier handelt es sich um eine Übersetzungsleistung, die schnell schief gehen kann, und daher einer gewissen Expertise in beiden Bereichen bedarf.

Braucht die Rechtswissenschaft also eine empirische Wende? - Sicherlich nicht in Form eines Paradigmenwechsels. Die Rechtswissenschaft ist und bleibt in erster Linie eine Normwissenschaft, die sich vor allem mit normativen Fragestellungen und Systembildung beschäftigt und ihr Instrumentarium entsprechend ausgebildet hat. ${ }^{95}$ Juristen können auch nicht gleichzeitig zu exzellenten Norminterpreten und guten Statistikern oder Experimentatoren ausgebildet werden die Investitionskosten wären regelmäßig zu hoch. ${ }^{96}$ Auf der anderen Seite ist es auch keine Option, die empirischen Fragestellungen einfach zu übergehen oder zu delegieren.

Aber es gibt nicht nur binäre entweder/oder-Lösungen. Vielmehr sollte es möglich sein, einen Mittelweg zu gehen. Die Rechtswissenschaft braucht zum einen Wissenschaftler, die eine gewisse Brückenfunktion ausüben und sich in mehr als nur der juristischen akademischen Welt auskennen. ${ }^{97}$ Deren Aufgabe ist es, Themen in Nachbarwissenschaften zu identifizieren, die Bedeutung für rechtswissenschaftliche Fragestellungen haben und eine Übersetzungsleistung zu erbringen, mit der diese Themen der Rechtswissenschaft näher gebracht und die Bedeutung für juristische Fragestellungen aufgezeigt wird. Zum anderen wäre es sicherlich nicht von Nachteil, jungen Juristen in der Ausbildung einen kleinen Kompass für empirische Fragestellungen mit auf den Weg zu geben. Das Studium konzentriert sich zur Zeit vollständig auf die normative Seite der Rechtswissenschaft. Von einem Umdenken in dieser Hinsicht profitierten auf lange Sicht Wissenschaft wie Praxis.

95 Armin von Bogdandy, Wissenschaft vom Verfassungsrecht: Vergleich, in: ders./Pedro Cruz Villalón/Peter Michael Huber (Hg.), Handbuch Ius Publicum Europaeum. Band II: Offene Staatlichkeit - Wissenschaft vom Verfassungsrecht, 2008, § 39, Rn. 31; Jestaedt (Fn. 23), 37.

96 Lüdemann (Fn. 69), 129.

97 In diese Richtung auch Andreas Voßkuhle, Methode und Pragmatik im Öffentlichen Recht, in: Hartmut Bauer/Detlef Czybulka/Wolfgang Kahl/Andreas Voßkuhle (Hg.), Umwelt, Wirtschaft und Recht. Wissenschaftliches Symposium aus Anlaß des 65. Geburtstages von Reiner Schmidt, 2002, 171, 194. 